\title{
Intracranial Pressure and the Role of the Vagus Nerve: A Hypothesis
}

\author{
Gardar Gudmundsson \\ Uppsalir Medical Center, Reykjavik, Iceland \\ Email: gardar@grusk.is
}

Received 15 March 2014; revised 15 April 2014; accepted 23 April 2014

Copyright (C) 2014 by author and Scientific Research Publishing Inc.

This work is licensed under the Creative Commons Attribution International License (CC BY).

http://creativecommons.org/licenses/by/4.0/

c) (i) Open Access

\begin{abstract}
There is reason to believe that the vagus nerve carries sensory afferent messages from the intracranial cavity as it does come from the other body cavities. Considering the arachnoid granulations as a secretory apparatus instead of an organ transporting cerebrospinal fluid, a simple model can be built. Assuming that the arachnoid granulations produce nitric oxide in accordance with changes in the intracranial pressure, the jugular foramen with the vagus nerve lying just subendothelially is where this message would be received. Other chemical substances from the granulations or other intracranial tissues could of course also act as messengers. This would mean that the brain stem could adjust to changes in intracranial pressure with small changes in cerebral venous resistance. The general idea is that intracranial pressure is a passive function of the intracranial pressure-volume relationship. But the hypothesis suggests that the vagus nerve constantly mediates changes in intracranial pressure to the brain stem that can rapidly adjust cerebral venous resistance. This control is totally intracranial and easily overridden by considerable changes in absorption of spinal fluid or intracranial calamities. This article looks for clues in support of the idea that the vagus nerve mediates intracranial pressure changes to the brain stem.
\end{abstract}

\section{Keywords}

Intracranial Pressure, Vagus Nerve, Nitric Oxide, Arachnoid Granulations, Jugular Foramen, Cerebrospinal Fluid

\section{Introduction}

Many authors have doubted the textbook description of cerebrospinal fluid (CSF) circulation, with the CSF being absorbed into the arachnoid granulations (AG) at the top of the cranial cavity and then secreted from vacuoles into the venous blood in the large sinuses [1]-[5]. 
In my 2013 article "Normal Pressure Hydrocephalus: A Simple Hypothesis” [6], I suggested that our idea of the circulation of the CSF was fundamentally incorrect regarding the issues of where the CSF absorption takes place. I suggested that the absorption mostly takes place in the subpial space (into subpial veins) under the brain and not in the AG, and that the AG are essentially of a sensory/secretory nature, probably secreting nitric oxide (NO) in accordance with changes in intracranial pressure (ICP).

These are extraordinary claims that need extraordinary proof, which I cannot provide, but searching through the literature gives indirect support to the hypothesis.

\section{Support for the Hypothesis}

The pressure-volume relationship within the skull, regarding the volumes of blood, brain and CSF is well known as the Monro-Kellie doctrine, which states that in the fixed intracranial cavity one component can only increase if others are decreased. The CSF production is more or less constant throughout life, and cerebral perfusion pressure (CPP) is controlled by autoregulation and the ICP is assumed to more or less rise and fall passively. The autoregulation controlled by the brain stem is not completely understood.

Blood flow through the brain is relatively constant and the heart and the arterial system can regulate flow to the cranial cavity to some degree by regulating actions of the heart and the tone in the vessel walls. The resistance in the cerebral veins is assumed to be influenced directly by the ICP [7].

The brain stem does not have full control over the intracranial blood-delivering functions of the heart and vascular system. Its control is only modulatory.

In an article from 1988 called "Cerebral Autoregulation Dynamics in Humans" by Aslid et al. in Oslo, Norway [8], the authors documented the response of cerebral blood flow to changes in arterial blood pressure and the effects of hypo-, normo-and hypercapnia in healthy volunteers. In their discussion, among other things, they pointed out that changes in head posture have a large impact on perfusion pressure, that autoregulation takes place in small vessels with a diameter $<40 \mu \mathrm{m}$, that the first response in autoregulation has an initial peak of 0.5 seconds, and that this is not a myogenic mechanism. They suggest that there may be a local release of a regulatory substance through some unknown pathway.

\subsection{Details of the Hypothesis}

The hypothesis has all the components for this unknown pathway: changes in ICP lead to changes in NO production by the endothelial tissue of the AG into the large venous sinuses; the vagus nerve receives the message, afferent sensory pathways bring the medulla oblongata nuclei the information and efferent pathways cause changes in the resistance in the cerebral venous system.

This is a short feedback loop that constantly adjusts to small fluctuations in the ICP to keep the optimal cerebral homeostasis; in fact with every change in head position there is an adaptation to be made.

This is built on the fact that small changes in the cerebral venous resistance lead to considerable ICP changes.

\subsection{The Vagus Nerve}

The vagus nerve is a major player in the regulation of bodily homeostasis. It has a motor part, parasympathetic efferents and sensory afferents. The brain stem receives the messages from the sensory afferents that originate in the abdominal and thoracic viscera, from baroreceptors in the aortic arch and chemoreceptors in the aortic bodies. They also receive general sensory inputs from the external ear, tympanic membrane, external auditory meatus, larynx, pharynx and esophagus. Over $80 \%$ of the fibers of the vagus nerve are afferent viscerosensory fibers [9].

In 1998 Maier et al. in Colorado summarized their research regarding the vagal nerve by saying that there was little doubt that the vagus was an important pathway by which immune stimulation signals the brain, and this was done by paraganglia cells releasing a variety of transmitters and molecules that evoke vagus nerve response [10].

The stimulation of the left vagal nerve in pigs had considerable effect on the ICP without bradycardia or changes in blood pressure, according to a study from 2004 [11]. The ICP reduction lasted for 15 - 35 minutes in the 8 pigs tested. The results point to the existence of a vagal circuit that is solely intracranial.

Vagus nerve stimulation for epilepsy has shown its value and there is ongoing research regarding vagal ef- 
fects on the spleen whereby inflammation may be halted [12]. The vagus nerve continues to surprise in its huge role in homeostasis.

\subsection{The Arachnoid Granulations}

The AG develop as we begin, as infants, to raise our head and then sit up and walk, indicating that while the skull is still with open sutures there is no need for feedback control of the ICP. It is hard to understand, according to our textbooks, that we don't need AG to eliminate CSF from the cranial cavity for the first 12 - 18 months of our lives, during a period that may have frequent hydrocephalus pathology and shunt problems.

The idea is that the AG has a pressure sensory/secreting anatomy, rather than a liquid transporting design.

"On microscopic evaluation, the protuberant masses were composed of a mixture of variably dense fibrous connective tissue containing numerous fibroblasts, scattered arachnoid cell nests, and an irregular network of small vessels and delicate endothelium-lined spaces, most prominent in the basal regions" [13].

The anatomy of the AG is surprisingly complex if its sole function is to transport CSF into the sinuses.

\subsection{Nitric Oxide}

Nitric oxide synthases (NOSs) synthesize the NO molecule of which there are three isoforms: endothelial, neuronal and inducible.

NO is an important and short-lived molecule that is a vasodilator, among other functions. It is a known parasympathetic neurotransmitter, well suited to carry information within the cerebral venous circulation. There is also considerable evidence that NO increases activity in the brain stem sites that promotes efferent vagal activity [14].

\subsection{The Jugular Foramen}

Why the jugular foramen? Bearing in mind that structure often defines function, why does the vagus nerve lie subendothelially in the jugular foramen, the leading exit of the cerebral venous system? In the advential layer of the jugular bulb there is a lining of nonchromaffin-staining paraganglian cells that make nerve connections with the glossopharyngeal and vagus nerves [15]. These cells have a well-defined chemoreceptor function [16].

There may be a difference in sides regarding the jugular foramen and number of arachnoid granulations, as there are side differences in the vagal nerves.

\subsection{The Cerebral Venous Circulation}

A small change in venous rheology leads to a big change in cerebral venous blood volume. There are many possible ways this could function.

Cerebral microcirculation begins where 30 - $70 \mu \mathrm{m}$ arterioles penetrate into the brain substance. It has been shown that blood flow characteristics are much more complex in this microvasculature than in the cerebral macrocirculation. At this level, plasma viscosity and reb blood corpuscle (RBC) aggregations are the main determinants of blood flow resistance. Leukocytes also play a major role as determinants of flow in the microcirculation. Fibrinogen is the most potent of the plasma proteins and causes the RBC to stick loosely together. It should not be forgotten that the cerebral temperature is elevated above the body core temperature and this might facilitate control in the cerebral microcirculation. The atrial natriuretic factor (ANF) is a molecule that might contribute to decreasing the vascular intracerebral sympathetic tone [17].

Both old and new research supports the idea that the cerebral circulation has neurovascular control regulating cerebral blood flow, not only macrocirculation but also microcirculation [18], making the brain stem able to influence resistance in the microcirculation.

\subsection{Possible Consequences}

What consequences does this system have? I assume that this is a delicate system and many things can go wrong. Increased ICP leads to increased NO production from the AG which in turn decreases cerebral venous resistance and allows ventricles to enlarge.

Less NO production would increase cerebral venous resistance and reduced the size of ventricles, given that all other parameters are even. 
Subarachnoidal hemorrhage (SAH) is a serious event and following it a sympathetic storm rages that completely overrides this system and autoregulatory capacity may be temporarily lost.

Scientists in China hypothesize in an article from 2013 that normalization of NO might have a positive influence on brain microcirculation following SAH [19]. Zheng and his team state that decreased bioavailability of endogenous NO plays an important part in the pathogenesis of cerebral vasospasm in rabbits. NO levels were lower in rabbits with SAH compared with a control group and their final conclusion is that the use of NO treatments after SAH might reduce the risk of poor outcomes. They focus only on the arterial side of the vasculature and find to their surprise that after SAH the NO levels increased over time. Here the NO control of ICP may be taking over again after the SAH effects subside. They point out that hypoxic preconditioning prevents reduction in NO after SAH.

I think the hypothesis also explains the gradual increase in size of ventricles in many SAH patients, as the AG resume their NO production.

In hydrocephalic patients with low-pressure hydrocephalus (also known as negative-pressure hydrocephalus and syndrome of inappropriately low-pressure acute hydrocephalus) there is an acute ventriculomegaly and inappropriately low ICP. The subarachnoidal space has been shown to be collapsed or there is loss of effective cortical subarachnoidal space [20]. Neck wrapping reduces cerebral venous outflow from the cranial cavity and thus also reduces CSF elimination, opening up the subarachnoidal space so that the arachnoid granulations can resume their NO production.

H. L. Rekate et al. say in an article from 2012: "Problems such as pseudotumor cerebri, shunt failure with nonresponsive ventricles, and negative-pressure hydrocephalus can be explained by assessing the ability of ventricular CSF to flow to the cortical subarachnoid space (CSAS) and the ability of this fluid to exit this compartment. Ventricular enlargement at the time of shunt failure implies a failure of flow between the ventricles and CSAS, implying that all patients who show this phenomenon are potential candidates for endoscopic third ventriculostomy (ETV)" [21].

The ETV opens a passage for the CSF into the subarachnoidal space.

It seems that when there is no or very little NO production, the system is not working and there is low resistance in the venous microcirculation and the ventricles enlarge, and it is logical to assume that a collapsed subarchnoidal space will stop the AG from producing NO.

The presence of slit ventricles is a situation where overdrainage has led to collapse of the ventricles. Here low ICP reduces NO production that results in increased venous resistance and ventricles become smaller.

The presence of pseudotumor cerebri (PC) (also called idiopathic or benign intracranial hypertension) fits into the hypothesis only if we assume that the vagus nerve receives misleading information that then leads to increased venous resistance in the brain parenchyma.

Here we might be seeing the common effects of two or more causes. For instance disturbed flow in the sinuses may reduce NO concentration, or venous endothelial injury may disrupt the message pathway in the jugular foramen or the wrong messenger may dominate, to name some possible factors.

Normal pressure hydrocephalus fits the hypothesis, with its somewhat raised ICP and enlarged ventricles. In my opinion [6] this is a disease where subpial veins play the leading role (Table 1).

\section{Discussion}

The idea that the AG produce a messenger regarding changes in ICP seems plausible and NO is the ideal messenger molecule. We have information, that an intracranial ICP adjustment system exists and also that there is a vagal-mediated ICP adjusting system without extracranial vagal effects.

As the NO molecule is short-lived and intracerebral production may not reach the jugular vein, secretion directly into the large sinuses is more efficient. Other molecules from the venous cerebral circulation such as cytokines or other intravasal molecules may play a role in giving information to the vagus at the jugular foramen. The vagal nerve has some side differences in its functions and the NO messenger system may also have different roles on each side of the head.

This hypothesis suggests a model that is analogous to the vagal systems in the other cavities.

The feedback system leads to changes in the venous cerebral circulation and thereby explains changes in the size of the ventricle system, other things being equal, as the brain tissue changes in volume.

Increased ICP will be met with less venous resistance, less volume filling the veins, and giving way according to the Monro-Kellie doctrine. 
Table 1. Vagal mediated changes in venous resistance with changes in ICP according to the hypothesis.

\begin{tabular}{|c|c|c|c|c|c|c|}
\hline & ICP & $\begin{array}{l}\text { NO } \\
\text { prod. }\end{array}$ & $\begin{array}{c}\text { Vagal } \\
\text { activity }\end{array}$ & $\begin{array}{l}\text { Venous } \\
\text { resistance }\end{array}$ & $\begin{array}{l}\text { Ventricle } \\
\text { size }\end{array}$ & Clinical comments \\
\hline Raised ICP & $\uparrow$ & $\uparrow$ & normal & $\downarrow$ & $\uparrow$ & $\begin{array}{l}\text { Venous resistance decreases } \\
\text { according to Monro-Kellie doctrine }\end{array}$ \\
\hline Low ICP & $\downarrow$ & $\downarrow$ & normal & $\uparrow$ & $\downarrow$ & e.g. iatrogen \\
\hline SAH & $\uparrow \uparrow$ & $\uparrow \uparrow$ & normal & $\uparrow \uparrow$ & $\downarrow$ & $\begin{array}{l}\text { Sympathetic overflow overwrites the } \\
\text { vagus-ICP system }\end{array}$ \\
\hline $\begin{array}{l}\text { Obliterated } \\
\text { CSAS }\end{array}$ & $\begin{array}{l}\text { AG: no } \\
\text { ICP registr. }\end{array}$ & none & normal & $\downarrow$ & $\uparrow$ & $\begin{array}{l}\text { No information reaches the vagus, } \\
\text { venous resistance is low }\end{array}$ \\
\hline Slit ventricles & $\downarrow \downarrow$ & $\downarrow \downarrow$ & normal & $\uparrow \uparrow$ & $\downarrow \downarrow$ & Due to overdrainage \\
\hline PC & $\uparrow \uparrow$ & ? & $\begin{array}{l}\text { disturbed } \\
\text { information }\end{array}$ & $\uparrow \uparrow$ & $\downarrow \downarrow$ & $\begin{array}{l}\text { Pseudotumor cerebri breaks the rule, } \\
\text { probably disturbed/wrong message to vagus }\end{array}$ \\
\hline NPH & $\uparrow$ & $\uparrow$ & normal & $\downarrow$ & $\uparrow$ & $\begin{array}{l}\text { Disturbed absorption, not frank } \\
\text { hydrocephalus. Gradual change in ventricle size }\end{array}$ \\
\hline
\end{tabular}

ICP (intracranial pressure), NO (nitric oxide), SAH (subarachnoidal hemorrhage), CSAS (cortical subarachnoid space), PC (pseudotumor cerebri), NPH (normal pressure hydrocephalus). An arrow pointing upwards stands for: increase in values, production, resistance to venous flow and increase in ventricle size. An arrow pointing downwards stands for a decrease in the above mentioned parameters.

If the hypothesis is proven right, we may have new opportunities to control ICP in critical situations. The ideal would be to administer NO through the nasal mucosa directly into the most frontal part of the sagittal sinus.

The hypothesis can of course only be proven in actual clinical situations.

\section{Disclosure}

The author reports no conflict of interest.

\section{Acknowledgements}

I would sincerely like to thank Dr Terry G. Lacy for her editing of the manuscript.

\section{References}

[1] Greitz, D. and Hannerz, J. (1996) A Proposed Model of Cerebrospinal Fluid Circulation: Observations with Radionuclide Cisterography. American Journal of Neuroradiology, 17, 431-438.

[2] Weller, R.O., Dujanda, E., Yow, H.-Y. and Carare, R.O. (2009) Lymphatic Drainage of the Brain and the PathoPhysiology of Neurological Disease. Acta Neurochirurgica, 117, 1-14.

[3] Bulat, M., Lupret, V., Oreskovic, D. and Klarica, M. (2008) Transventricular and Transpial Absorption of Cerebro-Spinal Fluid into Cerebral Microvessels. Collegium Antropologicum, 32, 43-50.

[4] Sakka, L., Coll, G. and Chazal, J. (2011) Review of the Literature: Anatomy and Physiology of Cerebrospinal Fluid. European Annals of Otorhinolaryngology, Head and Neck Diseases, 128, 309-316. http://dx.doi.org/10.1016/j.anorl.2011.03.002

[5] Bulat, M. and Klarica, M. (2011) Recent Insights into a New Hydrodynamics of the Cerebrospinal Fluid. Brain Research Reviews, 65, 99-112. http://dx.doi.org/10.1016/j.brainresrev.2010.08.002

[6] Gudmundsson, G. (2013) Normal Pressure Hydrocephalus: A Simple Hypothesis. Open Journal of Modern Neurosurgery, 3, 4-8.

[7] Cipolla, M.J. (2009) Chapter 5: Control of Cerebral Blood Flow. The Cerebral Circulation. Morgan \& Claypool Life Sciences.

[8] Aaslid, R., Lindegaard, K.F., Sorteberg, W. and Nornes, H. (1989) Cerebral Autoregulation Dynamics in Humans. Stroke, 20, 45-529. http://dx.doi.org/10.1161/01.STR.20.1.45

[9] Greenstein, B. and Greenstein, A. (2000) Color Atlas of Neuroscience, Neuroanatomy and Neurophysiology. Thieme Stuttgart, New York, 228-231.

[10] Maier, S.F., Goehler, L.E., Fleshner, M., Watkins, L.R. (1998) The Role of the Vagus Nerve in Cytokine-to-Brain 
Communication. Annals New York Academy of Sciences, 840, 289-300. http://dx.doi.org/10.1111/j.1749-6632.1998.tb09569.x

[11] Tubbs, R.S., Wellons III, J.C., Blount, J.P. and Oakes, W.J. (2004) Left-Sided Vagus Nerve Stimulation Decreases Intracranial Pressure without Resultant Bradycardia in the Pig: A Potential Therapeutic Modality for Humans. Child's Nervous System, 20, 309-312. http://dx.doi.org/10.1007/s00381-004-0947-x

[12] Koopman, Frieda A., Miljko, S., Grazio, S., Sokolovic, S., Tracey, K., Levine, Y., et al. (2012) Pilot Study of Stimulation of the Cholinergic Anti-Inflammatory Pathway with an Implantable Vagus Nerve Stimulation Device in Patients with Rheumatoid Arthritis. Arthritis \& Rheumatism, 64, 451 (Abstract).

[13] Leach, J.L., Jones, B.V., Tomasick, T.A., Stewart, C.A. and Balko, M.G. (1996) Normal Appearance of Arachnoid Granulations on Contrast-Enchanced CT and MR of the Brain: Differentiation from Dural Sinus Disease. American Journal of Neuroradiology, 17, 1523-1532.

[14] Chowdhardy, S. and Townend, J.N. (2001) Nitric Oxide and Hypertension: Not Just an Endothelium Derived Relaxing Factor! Journal of Human Hypertension, 15, 219-227. http://dx.doi.org/10.1038/sj.jhh.1001165

[15] Makiese, O., Chibbaro, S., Marsella, M., Huy, P.T.B. and George, B. (2012) Jugular Foramen Paragangliomas: Management, Outcome and Avoidance of Complications in a Series of 75 Cases. Neurosurgical Review, 35, 185-194. http://dx.doi.org/10.1007/s10143-011-0346-1

[16] Rao, A.B., Koeller, K.K. and Adair, C.F. (1999) Paragangliomas of the Head and Neck: Radiologic-Pathologic Correlation. RadioGraphics, 19, 1605-1632. http://dx.doi.org/10.1148/radiographics.19.6.g99no251605

[17] Baskurt, O.K., Hardeman, M.R., Rampling, M.W. and Meiselman, H.J. (2007) Handbook of Hemorheology and Hemodynamics. IOS Press, Amsterdam.

[18] Kulik, T., Kusano, Y., Aronhime, S., Sandler, A.L. and Winn, H.R. (2008) Regulation of Cerebral Vasculature in Normal and Ischemic Brain. Neuropharmacology, 55, 281-288. http://dx.doi.org/10.1016/j.neuropharm.2008.04.017

[19] Zheng, R., Quin, L., Li, S., Xu, K. and Geng, H. (2014) CT Perfusion-Derived Mean Transit Time of Cortical Brain Has a Negative Correlation with the Plasma Level of Nitric Oxide after Subarachnoid Hemorrhage. Acta Neurochirurgica, 156, 527-533. http://dx.doi.org/10.1007/s00701-013-1968-6

[20] Hamilton, M.G. and Price, A.V. (2012) Syndrome of Inappropriately Low-Pressure Acute Hydrocephalus (SILPAH). Hydrocephalus. Acta Neurochirurgica Supplementum, 113, 155-159.

[21] Rekate, H.L., Nadkarni, T.D. and Wallace, D. (2012) The Importance of the Cortical Subarachnoid Space in Understanding Hydrocephalus. Journal of Neurosurgery, 116, 1-11. 